\title{
Griffin Mound Site (41UR142) Faunal Analyses
}

\section{LeeAnna Schniebs}

Unknown

Follow this and additional works at: https://scholarworks.sfasu.edu/ita

Part of the American Material Culture Commons, Archaeological Anthropology Commons, Environmental Studies Commons, Other American Studies Commons, Other Arts and Humanities Commons, Other History of Art, Architecture, and Archaeology Commons, and the United States History Commons

Tell us how this article helped you.

This Article is brought to you for free and open access by the Center for Regional Heritage Research at SFA ScholarWorks. It has been accepted for inclusion in Index of Texas Archaeology: Open Access Gray Literature from the Lone Star State by an authorized editor of SFA ScholarWorks. For more information, please contact cdsscholarworks@sfasu.edu. 


\section{Griffin Mound Site (41UR142) Faunal Analyses}

Creative Commons License

(c) (i) (8)

This work is licensed under a Creative Commons Attribution-NonCommercial 4.0 International License 


\title{
GRIFFIN MOUND SITE (41UR142) FAUNAL ANALYSES
}

\author{
LeeAnna Schniebs
}

\section{INTRODUCTION}

The investigation of the Griffin Mound site (4IUR 142) in the Little Cypress Creek basin in Upshur County, Texas, yielded 394 faunal specimens with a total assemblage weight of I27.71 grams. This sum includes all turtle shell, antler, and bone fragments. Faunal material was recovered from the site surface, four shovel tests, and four units in a 2 x $2 \mathrm{~m}$ excavation at this Middle Caddoan site (see Nelson et al. 1996), consisting of midden deposits and a large storage pit feature. The following sections of this article discuss the methods employed in the faunal analysis, results of taxonomic identification and quantification, and distribution of these remains.

\section{METHODOLOGY}

All prehistoric vertebrate remains were inventoried and weighed, and Excel 5.0 for Windows was used to manipulate the generated faunal data. An Ohaus digital scale, Model CT600-S, was used to record bone weight. All fragments recovered were analyzed by the author, using comparative collections on loan from, or housed at, the Institute of Applied Sciences, Zooarchaeology Laboratory, University of North Texas, Denton, Texas. Occasional supplements were required, using conventional osteological keys such as Olsen (1964), Gilbert (1980), and Schmid (1972). Identifications were made to the most specific category possible depending on the condition of the bone and available comparative material. Only positive identifications resulted in the assignment of faunal elements to genus or species.

Standard zooarchaeological methods have been used, with first the animal bones inventoried and bagged by Archeological and Environmental Consultants (Austin and Pittsburg), then submitted to me for identification and quantification. Both unidentifiable and identifiable pieces were analyzed in similar fashion. That is, the same attributes were recorded: taxon, clement and portion of that element, anatomical location of the element, condition of the bone and any notes on age, taphonomy, burning or breakage patterns, and presence of modification, if applicable. Provenience information was also recorded.

Quantification of the assemblage is summarized as number of identified specimens per taxon (NISP) and as minimum number of individuals (MNI) for identified elements. MNI estimates were calculated according to the most frequently occurring element, based on symmetry and element portion (Munzel 1986). In the mammalian class, teeth were used whenever possible.

The faunal data tables in this article are standard species lists with the number of occurrences for each animal. Those specimens regarded as unidentifiable (those coded to only class) have been consolidated into two general categories. Elements of non-diagnostic skeletal value (unidentifiable fragments and long bone shafts, see Olsen [1964]), are coded in an indeterminate category by class and size range. For example, specimens counted as "mammal" are from indeterminate-size mammals, and "large mammal" refers to a dcer-size mammal. Recording these specimens in a size category cnables the most precise level of observation as the specimen allows. In small samples, taking note of weight and the size categories of non-diagnostic elements broadens the function of the bone assemblage. However, percentages referred to in this report are calculated by NISP rather than weight. 
Weights of specimens by lot number can be found in the faunal data inventory, on file with Archcological and Environmental Consultants.

\section{RESULTS}

The following section describes the vertebrate taxa recovered from the Griffin Mound site. 'Taxonomic classes identified include reptile, ave, and mammal (lagomorpha, rodentia, and artiodactyla). None of the faunal specimens are modified. Number of identified specimens (NISP) and MNI for each taxon are summarized in Table 1, as are weights for each taxon and percentages of site assemblage. The composition of anatomical elements can be found in Table 2 .

\section{Assemblage Composition}

\section{Class Reptilia}

Order Testudinata, Family Emydidae: Box turtle (Terrapene sp.) is represented by two shell fragments. One specimen was recovered from Feature 1 (an apparent storage pit feature, see Nelson et al. [1996]) in Unit 5, Level $8(80-90 \mathrm{~cm} \mathrm{bs})$. The second piece came from I evel $4(40-50 \mathrm{~cm}$ bs) in Unit 7. Box turtles, which are strictly a North American species, range widely over the eastern and central United States and into the Southwest, and they also occur in many parts of Mexico. These are dry-land turtles that close their shells tightly when danger threatens (Conant 1975). Both pieces are burned.

Order Testudinata (family indeterminate): Twenty shell fragments from unidentifiable turtle were recovered from several levels (Levels 2-5 [20-60 cm bs] and 7-10 [70-162 cm bs |) of all four units, including six pieces from Feature 1, and one fragment found in Shovel Test 4. All of the specimens are burned.

Class Aves

Order indeterminate: An unidentifiable large bird is represented by onc bone fragment recovercd from Shovel Test $1(0-20 \mathrm{~cm}$ bs). This specimen compares favorably to turkey, but because of fragmentation, a specific identification was not possible. Turkey occurs as wild fowl in open woodland environments (Robbins et al. 1983). This could be the remains of any one of a number of large game birds, but the Caddos were known to hunt turkey (Newcomb 1961).

\section{Class Mammalia}

Order Lagomorpha, Family Leporidae: Cottontail rabbit (Sylvilagus sp.) is represented by four specimens. They were recovered from Shovel Test 2 and Test Units 7 , 8 , and 6/7/8 in various levels, including one specimen from Feature 1. Three fragments are burned. Currently, two species of cottontail inhabit this part of Northeast Texas: the Eastern cottontail (Sylvilagus floridanus) prefers heavy brush, strips of forest with open areas, edges of swamps, and weed patches; swamp rabbit (Sylvilagus aquaticus) prefers swamps, marshes, and wet bottomlands (Burt and Grossenheider 1980). Osteologically, the swamp rabbit is the largest of the cottontails within its range (Davis 1978). Based on the fragmentary remains, a specific identification was not possible. A minimum of one individual was present at the site.

Order Rodentia, Family Geomyidae: Plains Pocket Gopher (Geomys bursarius) is represented by one element. This mandible was recovered from Level $6(60-70 \mathrm{~cm} \mathrm{bs})$ in 


\begin{tabular}{|c|c|c|c|c|c|}
\hline Scientific Name & Common Name & NISP & MNI & $\%$ of Site & Wt./g \\
\hline Vertebrata (indeterminate) & unidentifiable & 18 & & 4 & 1.75 \\
\hline Testudinae & turtle & 20 & & 4 & 2.65 \\
\hline Terrapene sp. & box turtle & 2 & 1 & 1 & 0.9 \\
\hline Aves (large) & lg. bird & 1 & 1 & 1 & 0.3 \\
\hline Mammalia & mammal & 8 & & 2 & 0.9 \\
\hline Mammalia (small) & sm. mammal & 6 & & 2 & 0.4 \\
\hline Mammalia (medium) & med. mammal & 1 & 1 & 1 & 0.7 \\
\hline Mammalia (large) & Ig. mammal & 278 & & 70 & 77.2 \\
\hline Sylvilagus sp. & cottontail & 4 & 1 & 1 & 0.7 \\
\hline Geomys bursarius & Plains pocket gopher & 1 & 1 & 1 & 0.7 \\
\hline Artiodactyla (medium) & deer-size artiodactyl & 18 & & 4 & 30 \\
\hline Odocoileus sp. & deer & 35 & 1 & 8 & 10.31 \\
\hline \multirow[t]{2}{*}{ Cervidae } & elk or deer (antler) & 2 & & 1 & 1.2 \\
\hline & TOTAL & 394 & & 100 & 127.71 \\
\hline
\end{tabular}

\begin{tabular}{|c|c|c|c|c|c|c|c|c|c|}
\hline \multirow{2}{*}{ Scientific Name } & \multirow{2}{*}{ Common Name } & \multicolumn{2}{|c|}{ Element } & \multirow[b]{2}{*}{ teeth } & \multirow[b]{2}{*}{ cranial } & \multirow[b]{2}{*}{ axial } & \multirow[b]{2}{*}{ I. bones } & \multirow[b]{2}{*}{$\mathrm{pod} / \mathrm{ph} \mathrm{x}$} & \multirow[b]{2}{*}{ other } \\
\hline & & unid & [teeth] & & & & & & \\
\hline Vertebrata (indeterminate) & unidentifiable & 15 & & & & & 3 & & \\
\hline Testudinae & turtle & & & & & & & & 20 \\
\hline Terrapene sp. & box turtle & & & & & & & & 2 \\
\hline Aves (large) & lg. bird & & & & & 1 & & & \\
\hline Mammalia & mammal & 8 & & & & & & & \\
\hline Mammalia (small) & sm. mammal & 6 & & & & & & & \\
\hline Mammalia (medium) & med. mammal & & & & & & 1 & & \\
\hline Mammalia (large) & lg. mammal & 232 & & & 3 & & 43 & & \\
\hline Sylvilagus sp. & cottontail & & & & 1 & 1 & 1 & 1 & \\
\hline Geomys bursarius & Plains pocket gopher & & [1] & & 1 & & & & \\
\hline Artiodactyla (medium) & deer-size artiodactyl & & & & & & 13 & 5 & \\
\hline Odocoileus sp. & deer & & & 35 & & & & & \\
\hline \multirow[t]{2}{*}{ Cervidae } & elk or deer (antler) & & & & & & & & 2 \\
\hline & TOTAL & 261 & [1] & 35 & 5 & 2 & 61 & 6 & 24 \\
\hline & & & & & & & & & \\
\hline \multicolumn{10}{|l|}{ NOTE: } \\
\hline \multicolumn{10}{|c|}{ "Teeth" inc. enamel fragments as well as complete teeth. Those in brackets were not included in site totals. } \\
\hline \multicolumn{10}{|c|}{ "Cranial" inc. skull elements, mandible, and maxilla fragments. } \\
\hline \multicolumn{10}{|c|}{ "Axial" inc. ribs, verts, pelves, and scapulae. } \\
\hline \multicolumn{10}{|c|}{ "Long Bones" inc. fragments as well as complete long bones. } \\
\hline \multicolumn{10}{|c|}{ "Podials \& phx" inc. extreme lower leg bones. } \\
\hline "Other" inc. turtle shell and & antler fragments. & & & & & & & & \\
\hline
\end{tabular}


Test Unit 8. The specimen is not burned. Preferring grassland, alfalfa fields, pastures, roadsides, and railroad rights-of-way (Burt and Grossenheider 1980), this is the only pocket gopher found in the vicinity. It is probably a modern intrusion.

Order Artiodactyla, Family Cervidae: Deer (Odocoileus sp.) is represented by 35 elements, comprised of complete teeth and tooth enamel fragments. Seven enamel fragments are burned. They were found in Shovel Test 4, and Test Units 5, 7, and 8, from Levels 1 through 8 (midden and feature contexts) as well as the surface. A minimum of one individual is represented, but age could not be determined because of fragmentation. White-tailed Deer (Odocoileus virginianus) is the only species that currently occupies the general area, are they are found in forests, swamps, and open brushy areas (Burt and Grossenheider 1980). Prehistorically, other species may have been present, but the elements found in this site assemblage are from smaller individuals. White-tailed deer are known for their small size, as compared to the larger mule deer of the western United States.

In addition to this quantity, the 18 medium artiodactyl bones most likely also represent deer. Although pronghorn is also categorized as a medium-sized artiodactyl, a specific identification cannot be made based on post-cranial bone fragments. But sincc no pronghorn tooth fragments were recovered, it is probably safe to assume that no pronghorn were present at the site. Both animals are similar in size, but pronghorn antelope are found in open prairies and sagebrush plains well to the west of the Little Cypress Creek basin (see Burt and Grossenheider 1980).

Medium artiodactyl (deer) is probably also represented in the unidentifiable large mammal category $(n=278)$. Large mammal bones were recovered from all levels of all squares, except for Test Unit 5, level $10(100-110 \mathrm{~cm}$ bs), and Test Unit 7, Level 8 (80$100 \mathrm{~cm}$ bs). 'Totals range from 1-17, and most of these fragments are burned $(n=201)$.

Family Cervidae is also represented by two antler fragments. They were recovered from Test Unit 5, Level $7(70-80 \mathrm{~cm}$ bs), and Test Unit 8, Level $2(20-30 \mathrm{~cm} \mathrm{bs})$. Both fragments are burned. These too are probably deer remains rather than elk.

Indeterminate

Only four percent of the Griffin Mound faunal assemblage is recorded as indeterminate vertebrate $(n=18)$. These bone fragments are indiscernible even at the class level.

A large portion of the assemblage was not identifiable to taxon, but was sorted into catcgories by size and class. Unidentifiable large mammal remains dominate the faunal sample $(n=278,70 \%)$. Indeterminate mammal $(n=8)$ and small mammal $(n=6)$ bone fragments are present, but occurrences are minimal. The single medium-sized mammal bone fragment compares favorably to fox, but a specific identification was not attempted due to its fragmentation and warping from burning. Red fox (Vulpes fulva) and gray fox (Urocyon cinereoargenteus) are current residents of the project area, and both are similar in size. The red fox prefers a mixture of forest and open country, while the gray fox can be found in chaparral, open forests, and rimrock country (Burt and Grossenheider 1980). However, the red fox has been introduced historically for purposes of sport at several localities in eastern and central Texas (Davis 1978). 


\section{Assemblage Condition}

In gencral, the faunal material from the Griffin Mound site is highly fragmented, explaining the low Identifiability rate. Taphonomic patterns are absent on 313 specimens (Table 3). Surface observations include exfoliation and abrasion. Seventy percent of the site sample is burned $(\mathrm{n}=278)$, probably a result of trash disposal. Summary of burned specimens can be found in Table 4, and the distribution of these burned remains can be found in Table 5.

In addition to weathering, spiral fracturing was recorded during the faunal analysis. Spiral fractures are the result of impact on bone, such as striking it with a hammerstone or breaking it on an anvil. It is a common, expedient technique used in tool manufacturing, bone processing, and refuse disposal. Usually associated with large mammal long bones, spiral fracturing can also occur during trampling, carnivore gnawing, or any other severe impacts not necessarily associated with human activity. Ten specimens are recorded as spirally fractured (Table 6). The remainder of the large mammal sample is angularly fractured, suggesting that the bone was broken when it was dry, rather than while green and fresh. Perhaps after processing, it was broken into smaller pieces for disposal.

Scavenging activities are minimal. Carnivore gnawing was observed on five specimens, while rodent gnawing was nonexistent (Table 6).

\section{DISCUSSION}

\section{Distribution}

The distribution of faunal remains within the midden and Feature 1 is summarized in Table 7. The following section organizes the Griffin Mound faunal collection according to recovery by shovel tests and test units. Specific recovery by unit and level can be found in Tables 8 through 11.

\section{Surface Collection and Shovel Tests 1 through 4}

Sixteen specimens were collected from the surface of the site, comprised of large mammal, medium artiodactyl, and deer bone fragments. Four shovel tests yielded a total of 24 faunal specimens, with the majority recovered from 60 to $80 \mathrm{~cm}$ bs in Shovel Test 4 $(n=12)$. These samples yielded a variety of animals: unidentifiable turtle, large bird, indeterminate mammal, large mammal, cottontail, medium artiodactyl, and deer. Twentythree pieces are burned.

\section{Test Unit 5}

Eleven levels in Test Unit 5 yielded 101 faunal specimens, and over $55 \%$ of the sample is comprised of large mammal remains $(n=56)$. The remainder of the sample consists of indeterminate vertebrate, turtle, box turtle, unidentifiable mammals, medium artiodactyl, deer, and cervid. Level $4(40-50 \mathrm{~cm}$ bs) yielded the majority of specimens $(\mathrm{n}=26)$, while only one fragment was found in Level $10(100-110 \mathrm{~cm} \mathrm{bs})$ in Feature 1 . Seventy-seven fragments are burned, and the majority of these pieces $(n=21)$ came from Level 8 ( $80-90 \mathrm{~cm}$ bs) in Feature 1 . 


\begin{tabular}{|l|l|r|r|r|}
\hline Table 3. Summary of Taphonomic Patterns on 41UR142 Faunal Specimens \\
\hline Scientific Name & Common Name & Taphonomy Type & \\
\hline & & 18 & & \\
\hline Vertebrata (indeterminate) & unidentifiable & 20 & & \\
\hline Testudinae & turtle & 2 & & \\
\hline Terrapene sp. & box turtle & 1 & & \\
\hline Aves (large) & Ig. bird & 5 & & \\
\hline Mammalia & mammal & 6 & & \\
\hline Mammalia (small) & sm. mammal & 1 & & \\
\hline Mammalia (medium) & med. mammal & 204 & 7 & \\
\hline Mammalia (large) & Ig. mammal & 4 & & \\
\hline Sylvilagus sp. & cottontail & 1 & & \\
\hline Geomys bursarius & Plains pocket gopher & 14 & & \\
\hline Artiodactyla (medium) & deer-size artiodactyl & 35 & & \\
\hline Odocoileus sp. & deer & 2 & & \\
\hline Cervidae & elk or deer (antler) & 313 & 7 & 74 \\
\hline
\end{tabular}

\begin{tabular}{|c|c|c|c|c|c|}
\hline \multirow[t]{2}{*}{ Scientific Name } & \multirow[t]{2}{*}{ Common Name } & \multicolumn{2}{|c|}{ Degree of Burning } & \multirow[b]{2}{*}{ black } & \multirow[b]{2}{*}{ white } \\
\hline & & not & charred & & \\
\hline Vertebrata (indeterminate) & unidentifiable & 1 & & 3 & 14 \\
\hline Testudinae & turtle & & & 1 & 19 \\
\hline Terrapene sp. & box turtle & & & & 2 \\
\hline Aves (large) & lg. bird & & & & 1 \\
\hline Mammalia & mammal & 3 & & & 5 \\
\hline Mammalia (small) & sm. mammal & & & & 6 \\
\hline Mammalia (medium) & med. mammal & & & & 1 \\
\hline Mammalia (large) & lg. mammal & 77 & 8 & 17 & 176 \\
\hline Sylvilagus sp. & cottontail & 1 & & & 3 \\
\hline Geomys bursarius & Plains pocket gopher & 1 & & & \\
\hline Artiodactyla (medium) & deer-size artiodactyl & 5 & & 1 & 12 \\
\hline Odocoileus sp. & deer & 28 & 7 & & \\
\hline \multirow[t]{2}{*}{ Cervidae } & elk or deer (antler) & & & & 2 \\
\hline & TOTAL & 116 & 15 & 22 & 241 \\
\hline
\end{tabular}




\begin{tabular}{|c|c|c|c|c|c|c|c|c|c|c|c|c|c|}
\hline Unit & Scientific Name & Common Name & Level No. & & & & & & & & & & \\
\hline \multicolumn{3}{|c|}{ Surface Collection and Shovel Tests } & surface & ST 1 & ST 2 & ST 4 & & & & & & & \\
\hline$(B=23)$ & & & & 0to20 & 20 to 40 & 60 to 80 & & & & & & & \\
\hline & Testudinae & turtle & & & & 4 & & & & & & & \\
\hline & Aves (large) & lg. bird & & 1 & & & & & & & & & \\
\hline & Mammalia & mammal & & 2 & & & & & & & & & \\
\hline & Mammalia (large) & Ig. mammal & 5 & 1 & 2 & 7 & & & & & & & \\
\hline & Sylvilagus sp. & cottontail & & & 1 & & & & & & & & \\
\hline & & TOTAL & 5 & 4 & 3 & 11 & & & & & & & \\
\hline TU 5 & & & Lev 1 & Lev 2 & Lev 3 & Lev 4 & Lev 5 & Lev 6 & Lev 7 & Lev 8 & Lev 9 & Lev 10 & Lev 11 \\
\hline \multirow[t]{10}{*}{$(B=77)$} & & & & & & & & & & $\mathrm{F}-1$ & $\mathrm{~F}-1$ & $\mathrm{~F}-1$ & $\mathrm{~F}-1$ \\
\hline & Vertebrata (indeterminate) & unidentifiable & & & 1 & 2 & & & 1 & 4 & & 1 & \\
\hline & Testudinae & turtle & & & 1 & 2 & & & & 1 & & & \\
\hline & Terrapene sp. & box turtle & & & & & & & & 1 & & & \\
\hline & Mammalia & mammal & & & 3 & & & & & & & & \\
\hline & Mammalia (medium) & med. mammal & & & & & & & & 1 & & & \\
\hline & Mammalia (large) & lg. mammal & 2 & 1 & 1 & 9 & 10 & 9 & 4 & 8 & 4 & & 4 \\
\hline & Odocoileus sp. & deer & & &. & & & & & 6 & & & \\
\hline & Cervidae & elk or deer (antler) & & & & & & & 1 & & & & \\
\hline & & TOTAL & 2 & 1 & 6 & 13 & 10 & 9 & 6 & 21 & 4 & 1 & 4 \\
\hline TU 6 & & & Lev 1 & Lev 2 & Lev 3 & Lev 4 & Lev 5 & Lev 6 & Lev 7 & Lev 8 & Lev 9 & & \\
\hline \multirow[t]{6}{*}{$(B=73)$} & & & & & & & & & & $\mathrm{F}-1$ & $F-1$ & & \\
\hline & Vertebrata (indeterminate) & unidentifiable & & & & & & 1 & & & 1 & & \\
\hline & Testudinae & turtle & & 2 & & & 1 & & & & 1 & & \\
\hline & Mammalia (large) & ig. mammal & 10 & 3 & 9 & 10 & 2 & 5 & 11 & 10 & 3. & & \\
\hline & Artiodactyla (medium) & deer-size artiodactyl & & & 1 & 1 & & & 1 & & 1 & & \\
\hline & & TOTAL & 10 & 5 & 10 & 11 & 3 & 6 & 12 & 10 & 6 & & \\
\hline
\end{tabular}


Table 5, cont.

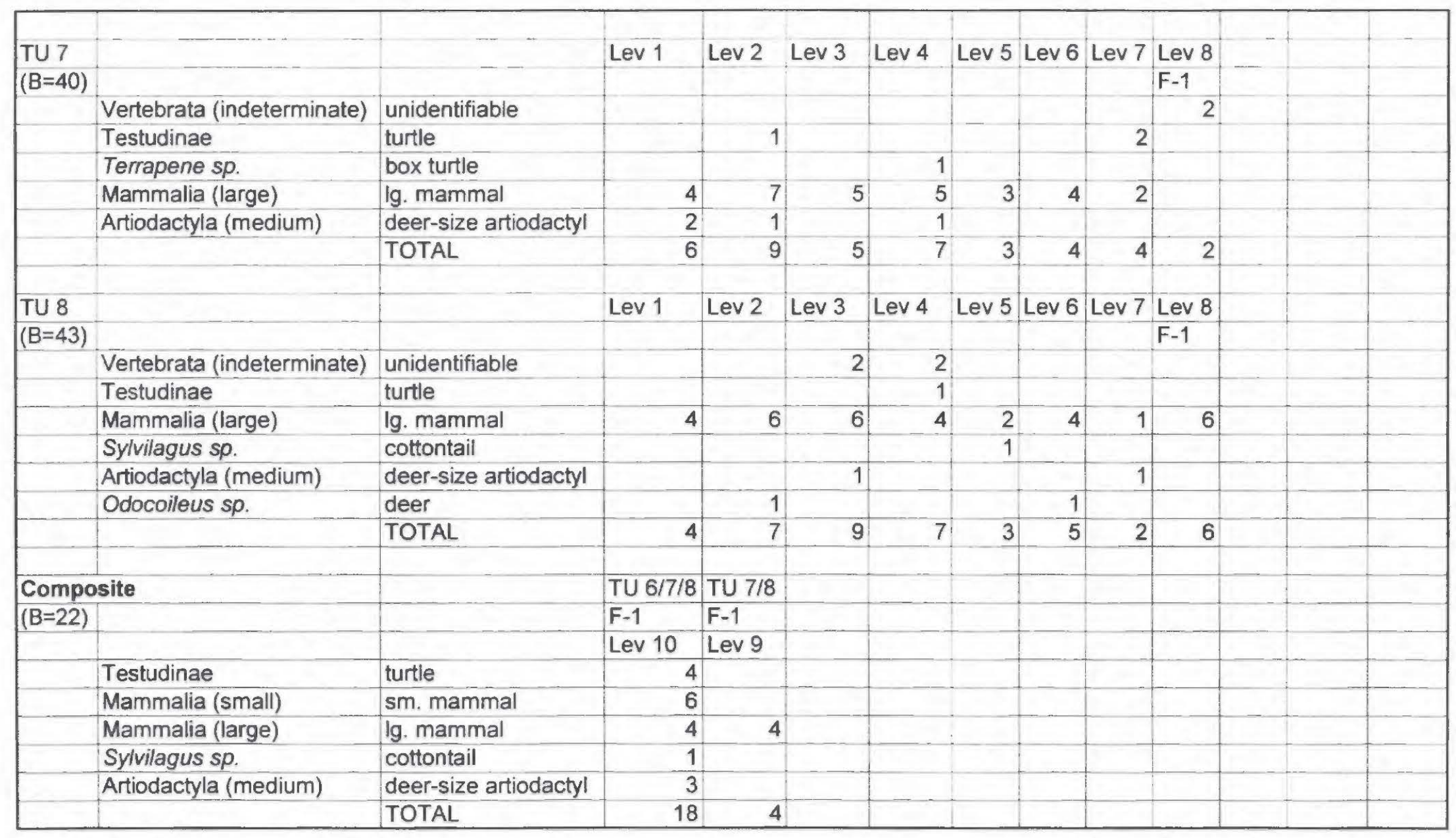




\begin{tabular}{|l|l|r|r|}
\hline Table 6. Summary of Gnawing and Spiral Fractures on 41UR142 Faunal Specimens \\
\hline Scientific Name & Common Name & carnivore gnawing & spiral fracture \\
\hline Mammalia (large) & Ig. mammal & 1 & 9 \\
\hline Artiodactyla (medium) & deer-size artiodactyl & 4 & 1 \\
& TOTAL & 5 & 10 \\
\hline
\end{tabular}

\begin{tabular}{|l|l|r|r|}
\hline Table 7. 41UR142 Faunal Recovery from Midden and Feature 1 \\
\hline Scientific Name & Common Name & Area & \\
\hline & & Midden & Feature 1 \\
\hline Vertebrata (indeterminate) & unidentifiable & 10 & 8 \\
\hline Testudinae & turtle & 14 & 6 \\
\hline Terrapene sp. & box turtle & 1 & 1 \\
\hline Aves (large) & Ig. bird & 1 & \\
\hline Mammalia & mammal & 8 & \\
\hline Mammalia (small) & sm. mammal & & \\
\hline Mammalia (medium) & med. mammal & & \\
\hline Mammalia (large) & Ig. mammal & 234 & 44 \\
\hline Sylvilagus sp. & cottontail & 3 & 1 \\
\hline Geomys bursarius & Plains pocket gopher & 1 & \\
\hline Artiodactyla (medium) & deer-size artiodactyl & 14 & 4 \\
\hline Odocoileus sp. & deer & 29 & 6 \\
\hline Cervidae & elk or deer (antler) & 2 & \\
\hline
\end{tabular}




\begin{tabular}{|c|c|c|c|c|c|c|c|c|c|c|c|c|}
\hline \multicolumn{13}{|c|}{ Table 8. Summary of 41UR142 Faunal Recovery by Unit } \\
\hline \multirow{2}{*}{ Scientific Name } & Common Name & Unit & & & & & & & & & & \\
\hline & & surface & ST 1 & ST 2 & ST 3 & ST 4 & TU 5 & TU 6 & TU 7 & TU 8 & TU $6 / 7 / 8$ & TU $7 / 8$ \\
\hline Vertebrata (indeterminate) & unidentifiable & & & & & & 9 & 2 & 3 & 4 & & \\
\hline Testudinae & turtle & & & & & 4 & 4 & 4 & 3 & 1 & 4 & \\
\hline Terrapene sp. & box turtle & & & & & & 1 & & 1 & & & \\
\hline Aves (large) & lg. bird & & 1 & & & & & & & & & \\
\hline Mammalia & mammal & & 2 & & 3 & & 3 & & & & & \\
\hline Mammalia (small) & sm. mammal & & & & & & & & & & 6 & \\
\hline Mammalia (medium) & med. mammal & & & & & & 1 & & & & & \\
\hline Mammalia (large) & Ig. mammal & 13 & 1 & 2 & 1 & 7 & 56 & 71 & 64 & 55 & 4 & 4 \\
\hline Sylvilagus sp. & cottontail & & & 1. & & & & & 1 & 1 & 1 & \\
\hline Geomys bursarius & Plains pocket gopher & & & & & & & & & 1 & & \\
\hline Artiodactyla (medium) & deer-size artiodactyl & 1. & 1 & & & & 1 & 5 & 4 & 3 & 3 & \\
\hline Odocoileus sp. & deer & 2 & & & & 1 & 25 & & 6 & 1 & & \\
\hline \multirow[t]{2}{*}{ Cervidae } & elk or deer (antler) & & & & & & 1 & & & 1 & & \\
\hline & TOTAL & 16 & 5 & 3 & 4 & 12 & 101 & 82 & 82 & 67 & 18 & 4 \\
\hline
\end{tabular}




\begin{tabular}{|c|c|c|c|c|c|c|c|}
\hline & & & & & & & \\
\hline & Scientific Name & Common Name & Unit & & & & \\
\hline Midden & & & & & & & \\
\hline Surface & & & surface & & & & \\
\hline & Mammalia (large) & lg. mammal & 13 & & & & \\
\hline & Artiodactyla (medium) & deer-size artiodactyl & 1 & & & & \\
\hline & Odocoileus sp. & deer & 2 & & & & \\
\hline & & TOTAL & 16 & & & & \\
\hline Level 1 & & & ST 1 & TU 5 & TU 6 & TU 7 & TU 8 \\
\hline & Aves (large) & Ig. bird & 1 & & & & \\
\hline & Mammalia & mammal & 2 & & & & \\
\hline & Mammalia (large) & Ig. mammal & 1 & 4 & 11 & 4 & 8 \\
\hline & Artiodactyla (medium) & deer-size artiodactyl & 1 & & & 2 & \\
\hline & Odocoileus sp. & deer & & 1 & & & \\
\hline & & TOTAL & 5 & 5 & 11 & 6 & 8 \\
\hline & & & & & & & \\
\hline Level 2 & & & ST 2 & TU 5 & TU 6 & TU 7 & TU 8 \\
\hline & Testudinae & turtle & & & 2 & 1 & \\
\hline & Mammalia (large) & Ig. mammal & 2 & 1 & 5 & 11 & 6 \\
\hline & Sylvilagus sp. & cottontail & 1 & & & 1 & \\
\hline & Artiodactyla (medium) & deer-size artiodactyl & & & & 1 & \\
\hline & Odocoileus sp. & deer & & 3 & & 1 & \\
\hline & Cervidae & elk or deer (antler) & & & & & 1 \\
\hline & & TOTAL & 3 & 4 & 7 & 15 & 7 \\
\hline & & & & & & & \\
\hline Level 3 & & & ST 3 & TU 5 & TU 6 & TU 7 & TU 8 \\
\hline & Vertebrata (indeterminate) & unidentifiable & & 1 & & 1 & 2 \\
\hline & Testudinae & turtle & & 1 & & & \\
\hline & Mammalia & mammal & 3 & 3 & & & \\
\hline & Mammalia (large) & Ig. mammal & 1 & 2 & 11. & 5 & 7 \\
\hline & Artiodactyla (medium) & deer-size artiodactyl & & 1 & 1 & & 1 \\
\hline & Odocoileus sp. & deer & & & & 1 & \\
\hline & & TOTAL & 4 & 8 & 12 & 7 & 10 \\
\hline
\end{tabular}


Table 9, cont.

\begin{tabular}{|c|c|c|c|c|c|c|c|}
\hline \multirow[t]{8}{*}{ Level 4} & & & ST 4 & TU 5 & TU 6 & TU 7 & TU 8 \\
\hline & Vertebrata (indeterminate) & unidentifiable & & 2 & & & 2 \\
\hline & Testudinae & turtle & 4 & 2 & & 1 & 1 \\
\hline & Terrapene sp. & box turtle & & & & 1 & \\
\hline & Mammalia (large) & Ig. mammal & 7 & 9 & 12 & 7 & 9 \\
\hline & Artiodactyla (medium) & deer-size artiodactyl & & & 1 & & 1 \\
\hline & Odocoileus sp. & deer & 1 & 13 & & 1 & \\
\hline & & TOTAL & 12 & 26 & 13 & 9 & 13 \\
\hline \multirow{6}{*}{ Level 5} & & & TU 5 & TU 6 & TU 7 & TU 8 & \\
\hline & Testudinae & turtle & & 1 & & & \\
\hline & Mammalia (large) & Ig. mammal & 10 & 3 & 5 & 14 & \\
\hline & Sylvilagus sp. & cottontail & & & & 1 & \\
\hline & Odocoileus sp. & deer & & & 1 & & \\
\hline & & TOTAL & 10 & 4 & 6 & 15 & \\
\hline \multirow{7}{*}{ Level 6} & & & & & & & \\
\hline & Vertebrata (indeterminate) & unidentifiable & 105 & TU 6 & $10 \%$ & 108 & \\
\hline & Mammalia (large) & Ig. mammal & 9 & 5 & 17 & 4 & \\
\hline & Geomys bursarius & Plains pocket gopher & & & & 1 & \\
\hline & Artiodactyla (medium) & deer-size artiodactyl & & 1. & & & \\
\hline & Odocoileus sp. & deer & 1 & & 1 & 1 & \\
\hline & & TOTAL & 10 & 7 & 18 & 6 & \\
\hline & & & & & & & \\
\hline \multirow[t]{8}{*}{ Level 7} & & & TU 5 & TU 6 & TU 7 & TU 8 & \\
\hline & Vertebrata (indeterminate) & unidentifiable & 1 & & & & \\
\hline & Testudinae & turtle & & & 2 & & \\
\hline & Mammalia (large) & lg. mammal & 4 & 11 & 15 & 1 & \\
\hline & Artiodactyla (medium) & deer-size artiodactyl & & 1 & & 1. & \\
\hline & Odocoileus sp. & deer & 1. & & 2 & & \\
\hline & Cervidae & elk or deer (antler) & 1 & & & & \\
\hline & & TOTAL & 7 & 12 & 19 & 2. & \\
\hline
\end{tabular}


Table 9, cont.

\begin{tabular}{|c|c|c|c|c|c|c|}
\hline \multicolumn{7}{|l|}{ Feature 1} \\
\hline \multirow[t]{8}{*}{ Level 8} & & & TU 5 & TU 6 & TU 7 & TU 8 \\
\hline & Vertebrata (indeterminate) & unidentifiable & 4 & & 2 & \\
\hline & Testudinae & turtle & 1 & & & \\
\hline & Terrapene sp. & box turtle & 1 & & & \\
\hline & Mammalia (medium) & med. mammal & 1 & & & \\
\hline & Mammalia (large) & Ig. mammal & 8 & 10 & & 6 \\
\hline & Odocoileus sp. & deer & 6 & & & \\
\hline & & TOTAL & 21 & 10 & 2 & 6 \\
\hline \multicolumn{7}{|l|}{ Level 9} \\
\hline & Vertebrata (indeterminate) & unidentifiable & TU 5 & TU 6 & TU $7 / 8$ & \\
\hline & Testudinae & turtle & & 1 & & \\
\hline & Terrapene sp. & box turtle & & 1 & & \\
\hline & Mammalia (large) & lg. mammal & 4 & 3 & 4 & \\
\hline & Artiodactyla (medium) & deer-size artiodactyl & & 1. & & \\
\hline & & TOTAL & 4 & 6 & 4. & \\
\hline \multirow[t]{8}{*}{ Level 10} & & & TU 5 & TU $6 / 7 / 8$ & & \\
\hline & Vertebrata (indeterminate) & unidentifiable & 1 & & & \\
\hline & Testudinae & turtle & & 4 & & \\
\hline & Mammalia (small) & sm. mammal & & 6 & & \\
\hline & Mammalia (large) & Ig. mammal & & 4. & & \\
\hline & Sylvilagus sp. & cottontail & & 1. & & \\
\hline & Artiodactyla (medium) & deer-size artiodactyl & & 3 & & \\
\hline & & TOTAL & 1. & 18 & & \\
\hline \multirow[t]{2}{*}{ Level 11} & & & TU 5 & & & \\
\hline & Mammalia (large) & lg. mammal & 5 & & & \\
\hline
\end{tabular}




\begin{tabular}{|c|c|c|c|c|c|c|c|c|c|c|c|c|c|}
\hline \multicolumn{14}{|c|}{ Table 10. Summary of 41UR142 Faunal Recovery by Level } \\
\hline & & & & & & & & & & & & & \\
\hline \multirow[t]{3}{*}{ Scientific Name } & Common Name & Level & & & & & & & & & & & \\
\hline & & surface & Lev 1 & Lev 2 & Lev 3 & Lev 4 & Lev 5 & 5 Lev 6 & Lev 7 & Lev 8 & Lev 9 & Lev 10 & Lev 11 \\
\hline & & & & & & & & & & F-1 & $\mathrm{F}-1$ & $\mathrm{~F}-1$ & $\mathrm{~F}-1$ \\
\hline Vertebrata (indeterminate) & unidentifiable & & & & 4 & 4 & & 1 & 1 & 6 & 1 & 1 & \\
\hline Testudinae & turtle & & & 3 & 1 & 7 & 1 & & 2 & 1 & 1 & 4 & \\
\hline Terrapene sp. & box turtle & & & & & 1 & & & & 1 & & & \\
\hline Aves (large) & lg. bird & & 1 & & & & & & & & & & \\
\hline Mammalia & mammal & & 2 & & 6 & & & & & & & & \\
\hline Mammalia (small) & sm. mammal & & & & & & & & & & & 6 & \\
\hline Mammalia (medium) & med. mammal & & & & & & & & & 1 & & & \\
\hline Mammalia (large) & lg. mammal & 13 & 28 & 25 & 26 & 44 & 32 & 35 & 31 & 24 & 11 & 4 & 5 \\
\hline Sylvilagus sp. & cottontail & & & 2 & & & 1 & & & & & 1 & \\
\hline Geomys bursarius & Plains pocket gopher & & & & & & & 1 & & & & & \\
\hline Artiodactyla (medium) & deer-size artiodactyl & 1 & 3 & 1 & 3 & 3 & & 1 & 2 & & 1 & 3 & \\
\hline Odocoileus sp. & deer & 2 & 1 & 4 & 1 & 14 & 1 & 3 & 3 & 6 & & & \\
\hline \multirow[t]{2}{*}{ Cervidae } & elk or deer (antler) & & & 1 & & & & & 1 & & & & \\
\hline & TOTAL & 16 & 35 & 36 & 41 & 73 & 35 & 41 & 40 & 39 & 14 & 19 & 5 \\
\hline
\end{tabular}




\begin{tabular}{|c|c|c|c|c|c|c|c|c|c|c|c|c|c|}
\hline \multicolumn{14}{|c|}{ Table 11. 41UR142 Faunal Recovery Unit Distribution } \\
\hline & & & \multirow{2}{*}{\multicolumn{2}{|c|}{ Level No. }} & & & & & & & & & \\
\hline Unit & Scientific Name & Common Name & & & & & & & & & & & \\
\hline & & & & & & & & & & & & & \\
\hline \multicolumn{2}{|c|}{ Surface Collection and Shovel Tests } & & surface & ST 1 & ST 2 & ST 3 & ST 4 & & & & & & \\
\hline \multirow[t]{10}{*}{$(N=40)$} & & & & Oto20 & 20 to 40 & 40 to60 & 60 to 80 & & & & & & \\
\hline & Testudinae & turtle & & & & & 4 & & & & & & \\
\hline & Aves (large) & lg. bird & & 1 & & & & & & & & & \\
\hline & Mammalia & mammal & & 2 & & 3. & & & & & & & \\
\hline & Mammalia (large) & Ig. mammal & 13 & 1 & 2 & 1 & 7 & & & & & & \\
\hline & Sylvilagus sp. & cottontail & & & 1 & & & & & & & & \\
\hline & Artiodactyla (medium) & deer-size artiodactyl & 1 & 1 & & & & & & & & & \\
\hline & Odocoileus sp. & deer & 2 & & & & 1 & & & & & & \\
\hline & & TOTAL & 16 & 5 & 3 & 4 & 12 & & & & & & \\
\hline & & & & & & & & & & & & & \\
\hline TU 5 & & & Lev 1 & Lev 2 & Lev 3 & Lev 4 & Lev 5 & Lev 6 & Lev 7 & Lev 8 & Lev 9 & Lev 10 & Lev 11 \\
\hline \multirow[t]{10}{*}{$(\mathrm{N}=101)$} & & & & & & & & & & $\mathrm{F}-1$ & F-1 & $F-1$ & F-1 \\
\hline & Vertebrata (indeterminate) & unidentifiable & & & 1 & 2 & & & 1 & 4 & & 1 & \\
\hline & Testudinae & turtle & & & 1 & 2 & & & & 1 & & & \\
\hline & Terrapene sp. & box turtle & & & & & & & & 1 & & & \\
\hline & Mammalia & mammal & & & 3 & & & & & & & & \\
\hline & Mammalia (medium) & med. mammal & & & & & & & & 1 & & & \\
\hline & Mammalia (large) & ig. mammal & 4 & 1 & 2 & 9 & 10 & 9 & 4 & 8 & 4 & & 5 \\
\hline & Odocoileus sp. & deer & 1 & 3 & 1 & 13 & & 1 & 1 & 6 & & & \\
\hline & Cervidae & elk or deer (antler) & & & & & & & 1 & & & & \\
\hline & & TOTAL & 5 & 4 & 8 & 26 & 10 & 10 & 7 & 21 & 4 & 1 & 5 \\
\hline TU 6 & & & Lev 1 & Lev 2 & Lev 3 & Lev 4 & Lev 5 & Lev 6 & Lev 7 & Lev 8 & Lev 9 & & \\
\hline$(N=82)$ & & & & & & & & & & $\mathrm{F}-1$ & F-1 & & \\
\hline & Vertebrata (indeterminate) & unidentifiable & & & & & & 1 & & & 1 & & \\
\hline & Testudinae & turtle & & 2 & & & 1 & & & & 1 & & \\
\hline & Mammalia (large) & lg. mammal & 11 & 5 & 11 & 12 & 3 & 5 & 11 & 10 & 3 & & \\
\hline & Artiodactyla (medium) & deer-size artiodactyl & & & 1 & 1 & & 1 & 1 & & 1 & & \\
\hline & & TOTAL & 11 & 7 & 12 & 13 & 4 & 7 & 12 & 10 & 6 & & \\
\hline
\end{tabular}


Table 11, cont.

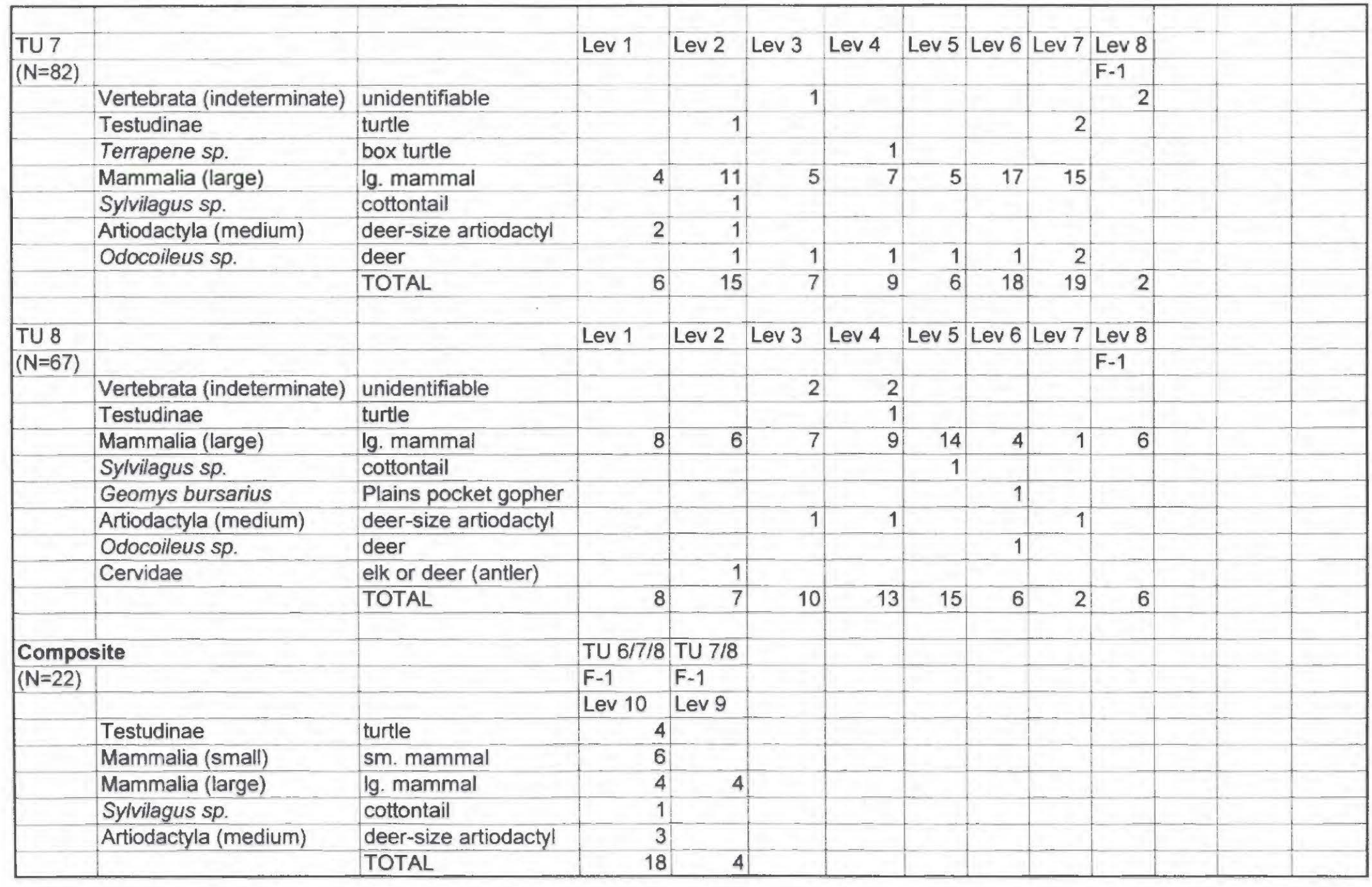




\section{Test Unit 6}

A total of 82 faunal specimens were recovered from nine levels in Test Unit 6. Again, large mammal dominates the sample $(n=71,87 \%)$, but indeterminate vertebrate, turtle, and medium artiodactyl bones are also present. The majority of bone fragments $(\mathrm{n}=13)$ came from Level $4(40-50 \mathrm{~cm}$ bs), but Levels $1(0-20 \mathrm{~cm}$ bs $), 3(30-40 \mathrm{~cm}$ bs $), 7$ $(70-80 \mathrm{~cm} \mathrm{bs}$ ), and 8 (Feature $1,80-100 \mathrm{~cm} \mathrm{bs)}$ yielded at least 10 specimens each. Seventy-three bone fragments from this unit are burned.

\section{Test Unit 7}

Eight levels in Test Unit 7 yielded a total of 82 faunal specimens, and $78 \%$ of this sample is large mammal $(n=64)$. The taxonomic composition is similar to that of Test Unit 6 , but box turtle, cottontail, and deer remains were also recovered. The majority of fragments came from Level $7(70-80 \mathrm{~cm}$ bs), but Levels $2(20-30 \mathrm{~cm} \mathrm{bs})$ and $6(60-70 \mathrm{~cm}$ bs), also in the midden, yielded relatively high quantities also. Forty specimens from this unit are burned, with totals from each level ranging from two to nine.

Test Unit 8

Eight levels in Test Unit 8 yielded 67 faunal specimens. This sample is also dominated by large mammal bone fragments $(n=55,82 \%)$. The remainder is comprised of indeterminate vertebrate, turtle, cottontail, pocket gopher, medium artiodactyl, deer, and cervid remains, although quantities for each are minimal. Totals from each level range from two to 15 bones. Sixty-four percent of the unit sample is burned $(n=43)$.

\section{Composite Units}

Eighteen faunal specimens were recovered from Level 10 in Test Unit 6/7/8, at the base of Feature 1 (120-162 cm bs), comprised of turtle, small and large mammal, cottontail, and medium artiodactyl. An additional four specimens came from Level 9 ( 100 $120 \mathrm{~cm}$ bs) in Test Unit 7/8. All of thesc pieces are burned.

\section{SUMMARY}

The midden at the Griffin Mound site (4IUR142) yielded the majority of faunal remains $(\mathrm{n}=317,80 \%)$. The storage pit feature had 77 fragments, including the only small mammal bones and the possible fox bone. Otherwise, faunal representation is similar in both areas. The distribution of fauna by level is also similar throughout the archeological deposit, with two exceptions. Level 4 had the highest recovery $(n=73)$, while Level 11 (110-130 cm bs in Unit 5) yielded only five specimens. The faunal collection from the Griffin Mound site indicates a high reliance on large game animals, supplemented with turtlc, bird, and small animals such as coltontail. The samplc is very typical Caddoan subsistence refuse.

\section{REFERENCES CITED}

Burt, W. H. and R. P. Grossenheider

1980 A Field Guide to the Mammals. Peterson Field Guide Series, edited by R. T. Peterson, 3rd edition. Houghton Mifflin Co., Boston. 
Conant, $\mathrm{R}$.

1975 A Field Guide to Reptiles and Amphibians of Eastern and Central North America. Peterson Field Guide Series, 2nd edition. Houghton Mifflin Co., Boston.

Davis, W. B.

1978 The Mammals of Texas. Bulletin No. 41. Tcxas Parks and Wildlife Department, Austin.

Gilbert, B. M.

1980 Mammalian Osteology. B. M. Gilbert, Publisher, Laramie, Wyoming.

Munzel, S.

1986 Quantitative Analysis and the Reconstruction of Site Patterning. Paper presented at the Vth International Conferencc of the International Council for ArchaeoZoology, Aug. 25-30, Bordeaux, France.

Nelson, B., M. Turner, and T. K. Perttula

1996 Archaeological Investigations at the Griffin Mound Site: A Middle Caddoan Sitc in Upshur County, Texas. The Cache, Collected Papers on Texas Archeology, No. 3:49-67. Office of the State Archeologist, Texas Historical Commission, Austin.

Newcomb, W. W., Jr.

1961 The Indians of Texas from Prehistoric to Modern Times. University of Texas Press, Austin.

Olsen, S. J.

1964 Mammal Remains from Archaeological Sites, Part I: Southeastern and Southwestern United States. Papers of the Peabody Museum of Archaeology and Ethnology 56(1). Peabody Museum of Archaeology and Ethnology, Harvard University, Cambridge.

Robbins, C. S., B. Bruun, and H. S. Zim

1983 A Guide to Field Identification: Birds of North America. Revised edition by Western Publishing, Racine, Wisconsin.

Schmid, E.

1972 Atlas of Animal Bones. Elsevier Publishing, Amsterdam. 